\title{
Deep Learning Brings New Era in Echocardiography
}

\author{
Tomoko Ishizu, MD, PhD
}

C ardiac sarcoidosis is rare but a potential life-threatening disease, ${ }^{1}$ and in the advanced stage, conduction block, ventricular tachyarrhythmia, myocardial dysfunction, and heart failure become progressively apparent. Idiopathic granulomatous inflammation of the heart,

Article p 87

characterized by non-caseating granulomas, results in irreversible scars. Prednisone or immunosuppressive therapy

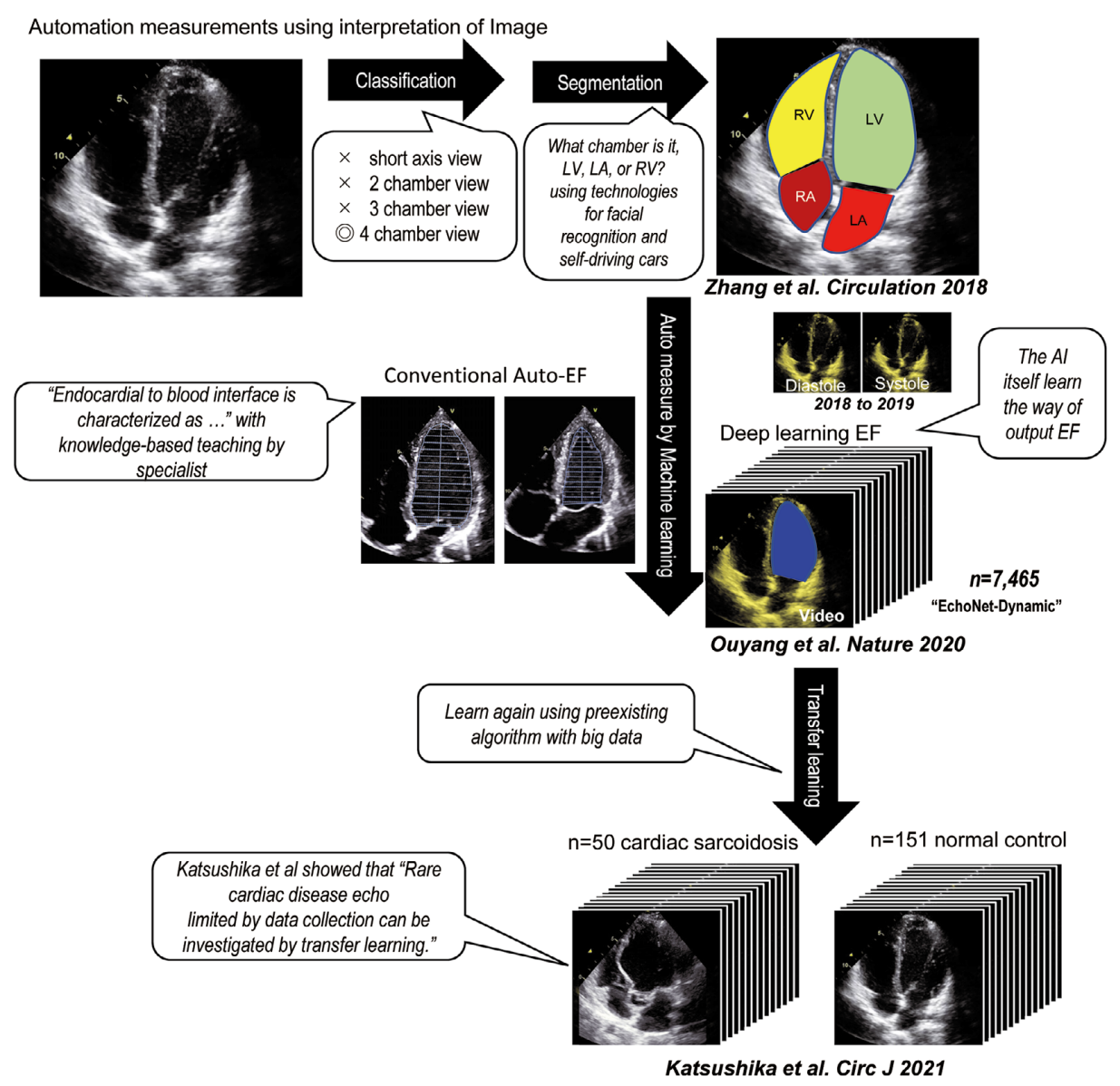

Figure. History of the application of artificial intelligence (AI) in interpreting echocardiographic images. EF, ejection fraction; LA, left atrium; LV, left ventricle; RV, right ventricle.

The opinions expressed in this article are not necessarily those of the editors or of the Japanese Circulation Society. Received July 26, 2021; accepted July 27, 2021; J-STAGE Advance Publication released online September 2, 2021

Department of Cardiology, Faculty of Medicine, University of Tsukuba, Tsukuba, Japan

Mailing address: Tomoko Ishizu, MD, PhD, Department of Cardiology, Faculty of Medicine, University of Tsukuba, 1-1-1 Tennodai,

Tsukuba 305-8575, Japan. E-mail: tomoco@md.tsukuba.ac.jp

All rights are reserved to the Japanese Circulation Society. For permissions, please e-mail: cj@j-circ.or.jp

ISSN-1346-9843 
can alter the clinical course if treatment begins before the point of no return. However, the diagnosis is often difficult and the low diagnostic accuracy may be due to the lack of specific imaging findings in this rare condition.

There are a lot of cardiac diseases that have low diagnostic accuracy, so if a new treatment strategy is developed, the differential diagnosis becomes critical. It often requires unique "red flag characteristics" found by specialists; for example, apical spearing in cardiac amyloidosis or annulus paradoxes in pericarditis. Sometimes, specialists can collect a limited number of cases, especially rare clinical entities.

In the daily cardiology clinic, echocardiography is the first-line diagnostic tool, mostly performed and interpreted by non-expert sonographers or cardiologists. Recently there has been a focus on artificial intelligence (AI) in echocardiography, initially intended to provide fundamental guidance for non-specialist users. Zhang et $\mathrm{al}^{2}$ reported on fully automated echocardiogram interpretation in 2018, using a computer vision algorithm that has been applied to facial recognition and self-driving cars, and they developed a method of identifying views (classification), delineating individual cardiac chambers (segmentation) (Figure, Upper), and providing the ejection fraction (EF) and global longitudinal strain with fair agreement with experts' measurements. It also offered differential diagnosis for hypertrophic cardiomyopathy, pulmonary hypertension, or cardiac amyloidosis from other clinical conditions. More recently, Ouyang et $\mathrm{al}^{3}$ reported echocardiography video-based AI that provides a video-based, not expert human tracing-based, conventional auto-EF algorithm "EchoNet-Dynamic", deep learning model for assessing the left ventricular EF on echocardiogram (Figure, Middle part). It predicted the EF with a small variance comparable to or less than that by human experts. Furthermore, reproducibility was superior to a human evaluation in real-time. It means that AI-derived EF prediction is not for only the non-expert anymore.

One of the challenging parts of developing a deep learning algorithm in echocardiography is the demand for big data. Both of the previous studies required 7,000 and $>14,000$ datasets to complete both echocardiographic and clinical information, respectively, to train, validate, and test the deep learning algorithms.

In this issue of the Journal, Katsushika et $\mathrm{al}^{4}$ report a new attempt to use a deep learning algorithm in echocardiographic movies of patients with cardiac sarcoidosis (Figure, Lower). They could collect only 151 echocardiog- raphy movies from 50 cardiac sarcoidosis patients over 5 years in a university hospital because of the rarity of this disease. They elegantly overcome the limitation of the number of patients by adapting the transfer learning of EchoNet-Dynamic. ${ }^{3}$ This methodology seems to have the potential to apply to other rare but important clinical disease entities. Their results showed comparable diagnostic ability of the deep learning algorithm with the cardiologist, even in patients with preserved left ventricular function.

As future research directions, multiple echocardiographic views and flow or color, or tissue Doppler echocardiography, speckle tracking echocardiography, and 3D echocardiography, and stress echocardiography could be good candidates for image assessment by AI. Furthermore, combined evaluation of multiple datasets and images using machine learning ${ }^{5}$ seems to be promising.

AI applications have initially focused on supporting non-expert data acquisition by classifying and segmenting the echocardiographic image. ${ }^{2}$ It initially dealt with still images, ${ }^{6}$ but has developed to analyze video images. ${ }^{3}$ Using a knowledge-based teaching algorithm to train a deep learning algorithm in which video is the only input data is a new way of analyzing limited available data, known as transfer learning. ${ }^{4}$

\section{References}

1. Tezuka D, Terashima M, Kato Y, Toriihara A, Hirasawa K, Sasaoka T, et al. Clinical characteristics of definite or suspected isolated cardiac sarcoidosis: Application of cardiac magnetic resonance imaging and $18 \mathrm{~F}$-fluoro-2-deoxyglucose positronemission tomography/computerized tomography. J Cardiac Fail 2015; 21: 313-322.

2. Zhang J, Gajjala S, Agrawal P, Tison GH, Hallock LA, Beussink-Nelson L, et al. Fully automated echocardiogram interpretation in clinical practice. Circulation 2018; 138: 1623-1635.

3. Ouyang D, He B, Ghorbani A, Yuan N, Ebinger J, Langlotz CP, et al. Video-based AI for beat-to-beat assessment of cardiac function. Nature 2020; 580: 252-256.

4. Katsushika S, Kodera S, Nakamoto M, Ninomiya K, Kakuda $\mathrm{N}$, Shinohara H, et al. Deep learning algorithm to detect cardiac sarcoidosis from echocardiographic movies. Circ J 2022; 86: $87-95$.

5. Kagiyama N, Piccirilli M, Yanamala N, Shrestha S, Farjo PD, Casaclang-Verzosa $\mathrm{G}$, et al. Machine learning assessment of left ventricular diastolic function based on electrocardiographic features. J Am Coll Cardiol 2020; 76: 930-941.

6. Kusunose K, Abe T, Haga A, Fukuda D, Yamada H, Harada $\mathrm{M}$, et al. A Deep learning approach for assessment of regional wall motion abnormality from echocardiographic images. JACC Cardiovasc Imaging 2020; 13: 374-381. 